\title{
Embodied Precision: Intranasal Oxytocin Modulates Multisensory Integration
}

\author{
Laura Crucianelli $^{1,2}$, Yannis Paloyelis ${ }^{3}$, Lucia Ricciardi ${ }^{4}$, \\ Paul M. Jenkinson ${ }^{2}$, and Aikaterini Fotopoulou ${ }^{1}$
}

\begin{abstract}
Multisensory integration processes are fundamental to our sense of self as embodied beings. Bodily illusions, such as the rubber hand illusion (RHI) and the size-weight illusion (SWI), allow us to investigate how the brain resolves conflicting multisensory evidence during perceptual inference in relation to different facets of body representation. In the RHI, synchronous tactile stimulation of a participant's hidden hand and a visible rubber hand creates illusory body ownership; in the SWI, the perceived size of the body can modulate the estimated weight of external objects. According to Bayesian models, such illusions arise as an attempt to explain the causes of multisensory perception and may reflect the attenuation of somatosensory precision, which is required to resolve perceptual hypotheses about conflicting multisensory input. Recent hypotheses propose that the precision of sensorimotor representations is
\end{abstract}

\section{INTRODUCTION}

When we wake up in the morning, we do not question whether our body belongs to us. However, the ability to recognize our body as our own (i.e., sense of body ownership) and related aspects of our coherent, mental representation of our body need to be learned (Blanke, 2012; Gallagher, 2000). Body image involves a conscious process to identify the body as our own, and therefore, it is closely related to our sense of body ownership. It includes visual perceptions and beliefs about our own body, and it is considered to be a fundamental aspect of bodily self-consciousness (see Blanke, 2012, for a review; Dijkerman, 2015).

Experimental methods of multisensory integration allow us to investigate these distinct facets of body representation. For example, the development of experimental paradigms that allow the controlled manipulation of limb ownership in laboratory settings, such as the rubber hand illusion (RHI; Botvinick \& Cohen, 1998), provides a unique tool to investigate the sense of body ownership. In this illusion, synchronous touch between

\footnotetext{
${ }^{1}$ University College London, ${ }^{2}$ University of Hertfordshire,

${ }^{3}$ King's College London, ${ }^{4}$ St. George's University of London

determined by modulators of synaptic gain, like dopamine, acetylcholine, and oxytocin. However, these neuromodulatory hypotheses have not been tested in the context of embodied multisensory integration. The present, double-blind, placebocontrolled, crossover study ( $n=41$ healthy volunteers) aimed to investigate the effect of intranasal oxytocin (IN-OT) on multisensory integration processes, tested by means of the RHI and the SWI. Results showed that IN-OT enhanced the subjective feeling of ownership in the RHI, only when synchronous tactile stimulation was involved. Furthermore, IN-OT increased an embodied version of the SWI (quantified as estimation error during a weight estimation task). These findings suggest that oxytocin might modulate processes of visuotactile multisensory integration by increasing the precision of top-down signals against bottom-up sensory input.

a visible rubber hand and the participant's hidden hand produces the illusion of ownership over the fake hand. This feeling of ownership toward the rubber hand arises as a solution to the unlikely conflict between sensory signals from three modalities that need to be integratedvision, touch, and proprioception (i.e., synchronous vision and touch but incongruent proprioception). People select the most plausible cross-modal hypothesis for the causes of these different sensations, that is, "The (rubber) hand I see being touched (vicarious touch) in synchrony with my own hand (felt via proprioception and epistemically private touch) is most likely to be mine" (Apps \& Tsakiris, 2014; Tsakiris \& Haggard, 2005). This hypothesis is more plausible than the hypothesis that this arm belongs to someone else, because I have the prediction that a hand-like stimulus seen in peripersonal space and from a first-person perspective is my own hand. Hence, greater weight is placed to visual signals (i.e., "where I see the rubber hand to be") relatively to the incompatible proprioceptive signals (i.e., "where I feel my own hand to be"). Because of the same embodied history, this prediction is even more likely when the hand I see is touched at the same time as the hand I feel and less likely when the two touches are asynchronous or spatially incompatible (Panagiotopoulou, Filippetti, 
Tsakiris, \& Fotopoulou, 2017; Abdulkarim \& Ehrsson, 2016; Ferri, Chiarelli, Merla, Gallese, \& Costantini, 2013). In the latter case, greater weight is placed to visual signals (i.e., "where and how I see the rubber hand to be touched") relatively to the incompatible somatosensory signals, comprising both proprioceptive signals (i.e., "where I feel my own hand to be") and tactile signals (i.e., "where and how I feel the touch to be in an epistemically private manner"). Recent studies have further shown that, when proprioception or somatosensation are damaged or become unreliable, this inferential process continues to the point that mere vision of a rubber hand in peripersonal space is sufficient to create strong feelings of rubber hand ownership, even without tactile stimulation (a phenomenon we have previously termed "visual capture of ownership"; Martinaud, Besharati, Jenkinson, \& Fotopoulou, 2017; see also Samad, Chung, \& Shams, 2015; Farnè, Pavani, Meneghello, \& Ladavas, 2000; Pavani, Spence, \& Driver, 2000). Taken together, RHI studies in healthy and clinical populations suggest that the sense of body ownership arises as a consequence of the attempt to find the most likely cause of multisensory signals, depending on their reliability (Apps \& Tsakiris, 2014; Zeller, Litvak, Friston, \& Classen, 2014).

The experience of the RHI can affect both the representation of our own body as well as the representation of the material and physical world beyond our body, for example, the perception of objects (Haggard \& Jundi, 2009). By means of an RHI paradigm, Haggard and Jundi (2009) manipulated body image representation, first explicitly, by inducing participants to embody either a larger or smaller rubber hand than their own hand. Participants were equally able to embody the larger and smaller rubber hand. Second, they tested whether participants were able to judge the weight of external objects having a constant size but different weights, which leads to an embodied version of the size-weight illusion (SWI; Haggard \& Jundi, 2009; Cesari \& Newell, 1999). Typically, in such illusions, the size of an object will influence our expectations of how heavy this object is (Flanagan \& Beltzner, 2000). In this embodied version of the illusion, the size of the object itself is not varied, but by varying the size of the embodied hand, any changes in the perception of the object weight are considered a result of the relative size of the object in comparison with the participants' "own" hand (Haggard \& Jundi, 2009). The results revealed that, although there was no effect of hand size on explicit feelings of embodiment, the represented size of the participants' hand had an impact on the perceived size and weight of the grasped object, inducing an SWI. In particular, only when participants acquired ownership of a larger hand, they perceived the grasped objects as smaller in size and therefore heavier in weight. This phenomenon can be explained by a violation of perceptual experience due to a mismatch between what we expect (e.g., the size of the object) and what we experience with our senses (e.g., the weight of the object; Haggard \& Jundi, 2009; Flanagan \& Beltzner, 2000). Interestingly, the SWI can be considered as an indirect measure of body size representation, in the sense that an induced variation in the perceived body size can be measured indirectly, that is, not by asking participants but by measuring the modulation of object weight perception. Hence, including this measure can reveal whether body representation is affected differently at an explicit versus implicit level.

These multisensory integration phenomena appear to relate to certain psychometric characteristics, such as eating disorders symptomatology and self-objectification. Indeed, people with eating disorders seem to be particularly susceptible to bodily illusions, in the sense that they score higher than controls during both synchronous and asynchronous conditions of tactile stimulation of their own and a fake, or virtual, body for example (Eshkevari, Rieger, Longo, Haggard, \& Treasure, 2012). This susceptibility persists even after otherwise successful recovery (Eshkevari, Rieger, Longo, Haggard, \& Treasure, 2014), suggesting a stable trait that is not the mere result of malnutrition at the acute stage of the illness as some other cognitive biases have been found to be (e.g., novelty seeking or emotion recognition; Treasure \& Schmidt, 2013; Wagner et al., 2006). In addition, the fact that people with eating disorders in comparison with healthy controls show greater proprioceptive drift and report stronger feelings of ownership over a fake hand irrespective of whether their own body is touched synchronously or asynchronously suggests that they tend to attribute greater weight to the visual information of a realistic hand in front of them and less weight to correspondence of this visual information with epistemically private proprioceptive signals and somatosensory signals about the felt touch. Recent studies suggest that (subclinical) eating disorder symptomatology might relate to body satisfaction after manipulation of illusory body size (Preston \& Ehrsson, 2014), suggesting that this measure might influence body size representation in healthy people. Similarly, the extent to which individuals experience their own body as an object to be evaluated based on its appearance rather than effectiveness (i.e., self-objectification) is related to eating disorder symptomatology and seems to affect body shame, anxiety, and eating disorders as well as being a potential precursor to depression and sexual dysfunction (Fredrickson \& Roberts, 1997). In addition, self-objectification accounts for the relative insensitivity of women to their own internal bodily cues, which has been reported in studies of interoception (Ainley \& Tsakiris, 2013; Tiggemann \& Lynch, 2001).

Computational approaches to multisensory integration illusions (Ernst \& Banks, 2002) such as the RHI (Samad et al., 2015) and the SWI (e.g., Buckingham \& Goodale, 2013) have shown that cross-modal conflicts are resolved in a Bayes-optimal manner, by weighting the various sensory signals with an appropriate level of confidence or 
precision (Zeller, Friston, \& Classen, 2016; O'Reilly, Jbabdi, \& Behrens, 2012). This concept is central to predictive coding theories of perception, which claim that the precision weighting of ambiguous or noisy sensory signals determines how they are used to update predictions about their sources (Friston, 2008; Friston \& Stephan, 2007). Specifically, predictive coding is based on the idea that the brain interprets sensory information according to a hierarchical generative model of the world, which generates top-down representations, against which bottom-up sensory signals are tested to update beliefs about their causes (e.g., Limanowski \& Blankenburg, 2015; Friston, 2005; Rao \& Ballard, 1999). In this context, perception can be considered as the process of minimizing prediction errors (i.e., difference between predictions and sensory signals) by forming Bayesoptimal, top-down predictions. Crucially, the balance and reciprocal influence between bottom-up signals and top-down predictions depends on their relative uncertainty (or formally its inverse, precision; Feldman \& Friston, 2010) or their reliability (Deroy, Spence, \& Noppeney, 2016; Rohe \& Noppeney, 2015). The predictive coding architecture we have in mind-to explain multisensory integration in this context-considers ascending prediction errors from multiple modalities that are all competing to update posterior beliefs of an amodal sort. The basic idea here is that, if these sources of sensory evidence are in conflict, the ensuing uncertainty can be resolved by downregulating incongruent or incompatible sources of information (e.g., the touch I see vs. the touch I feel; where I see the hand vs. where I feel my hand to be). Technically, according to predictive processing accounts, this corresponds to a decrease in the precision of incongruent prediction errors, relative to consistent and congruent prediction errors. In the experimental setting described below, this form of multisensory integration generally favors visual modalities-at the expense of proprioceptive and somatosensory import-in terms of providing a coherent explanation for the sensory evidence at hand.

For example, using modeling under a predictive coding framework, Zeller and colleagues suggested that, to resolve the uncertainty between the conflicting visual, proprioceptive, and tactile information in the RHI, the brain downregulates the precision of ascending somatosensory prediction errors (Zeller et al., 2014, 2016). In support of their prediction, Zeller et al. (2014) found that touch-evoked EEG potentials elicited by brush strokes during the RHI are selectively attenuated. These results are consistent with the idea that multisensory integration requires precision weighting of sensations according to their reliability, such that, in the RHI, the precision of somatosensory signals (i.e., felt touch and/or proprioceptive signals from the participant's own hand) needs to be attenuated relative to the precision of visual signals to resolve conflicting perceptual hypotheses about the most likely cause of sensations in favor of the visually per- ceived rubber hand (Zeller et al., 2014, 2016). Similarly, predictive coding models of the SWI suggest that this illusion is the product of perceptual processes specialized for the detection and monitoring of outliers in the environment with the final goal of efficient coding of information (Buckingham \& Goodale, 2013). In Bayesian terms, our weight perception is driven by our expectations of how heavy something should be. These priors are weighted against bottom-up signals and adjusted by the presence of lifting errors. When lifting an object for the first time, participants will apply either excessive or insufficient force; however, in subsequent trials, it is likely that previous experience will reduce the amount or size of such errors (Buckingham \& Goodale, 2013).

These Bayesian accounts of multisensory integration seem to suggest that the brain is able to represent and use estimates of uncertainty (more formally precision, namely, confidence or the inverse of uncertainty; Friston, 2010; Knill \& Pouget, 2004) to achieve an optimal coding of information, even if the underlying neural coding principles remain debated (e.g., Fiser, Berkes, Orbán, \& Lengyel, 2010 vs. Ma, Beck, Latham, \& Pouget, 2006). According to one view, precision is thought to be mediated by the gain or excitability of (superficial pyramidal) cells encoding prediction errors (Shipp, Adams, \& Friston, 2013; Bastos et al., 2012; Feldman \& Friston, 2010). Thus, optimizing precision corresponds to neuromodulatory gain control of neuronal populations reporting prediction error (Feldman \& Friston, 2010). On this hypothesis, modulators of synaptic gain (like dopamine, acetylcholine, and norepinephrine as well as neuropeptides such as oxytocin; Quattrocki \& Friston, 2014) therefore might play a role in determining the precision of sensorimotor representations encoded by the activity of the synapses they modulate (Friston, Adams, Perrinet, \& Breakspear, 2012; Yu \& Dayan, 2005; Fiorillo, Tobler, \& Schultz, 2003). However, to our knowledge, these hypotheses have not been tested in multisensory integration research. This study specifically aimed to study the role of the neuropeptide oxytocin on multisensory integration and, particularly, body ownership and body image representation.

Oxytocin is a neuropeptide consisting of nine amino acids, mostly synthesized in the hypothalamus. This neuropeptide acts peripherally as a hormone, such as when it is released during labor and breastfeeding to stimulate uterine contractions and milk ejection, respectively (Burbach, Young, \& Russell, 2006). Oxytocin also acts centrally as a neuromodulator (Grinevich, Knobloch-Bollmann, Eliava, Busnelli, \& Chini, 2016; UvnäsMoberg, Handlin, \& Petersson, 2015; Stoop, 2012). It has been proposed that oxytocin might modulate the relationship between sensory information and attentional biases by enhancing the precision of socially relevant information and attenuating the precision of nonsocial stimuli (Quattrocki \& Friston, 2014; Gordon et al., 2013). Oxytocin might interact with the dopaminergic system 
to increase the attention orientation toward social cues (Shamay-Tsoory \& Abu-Akel, 2016). The use of nasal sprays has provided a safe and noninvasive route for the administration of oxytocin to target the human brain (Paloyelis, Krahé, et al., 2016; MacDonald et al., 2011; Born et al., 2002). An increasing number of studies have proposed that intranasal oxytocin (IN-OT) can modulate social cognition, affiliation, and brain function in humans (Colonnello \& Heinrichs, 2016; UvnäsMoberg et al., 2015; Rocchetti et al., 2014; De Dreu et al., 2010; MacDonald \& MacDonald, 2010).

Here, in a parsimonious psychophysical setting, we examined whether (1) IN-OT affects body ownership during a classic RHI and (2) IN-OT modulates body image representation, by means of an enhanced version of the illusion combining the RHI with hands of different sizes and an SWI (as in Haggard \& Jundi, 2009). In both instances, individuals had to weigh conflicting sensations derived from the self (somatosensory signals) or rubber hands of different sizes (visual signals). Specifically, on the basis of theoretical proposals about the role of oxytocin on enhancing somatosensory attenuation (Quattrocki \& Friston, 2014), we hypothesized that IN-OT would enhance the embodiment of the rubber hand in the standard and in the enhanced RHI paradigm, by attenuating the precision of somatosensory signals (epistemically private felt touch) relative to the precision of visual signals (vicarious touch on the rubber hand). In the standard RHI paradigm (1), we predicted that IN-OT would modulate multisensory integration and corresponding subjective (i.e., self-report measure) and behavioral (i.e., the degree to which participants erroneously perceived changes in the position of their own, unseen hand toward the rubber hand, the so-called proprioceptive drift) measures of body ownership over the RHI. We expected to observe these effects in both mere visual capture conditions and during synchronous tactile stimulation of the rubber hand and participants' own hand. In the SWI combined with the RHI (2), we predicted that IN-OT would have an effect on body image representation by increasing the weight estimation error after embodiment of the larger hand; in contrast, no effect on weight estimation was expected after embodiment of the normal-sized hand.

In summary, we conducted a double-blind, placebocontrolled, crossover study to investigate the effect of a single dose of IN-OT compared with placebo on multisensory integration during a standard and an enhanced version of the RHI paradigm where we manipulated the synchronicity of touch (synchronous vs. asynchronous) and the size of the rubber hand (normal vs. large). We recorded subjective and behavioral measures of body ownership in both mere visual capture conditions and during synchronous tactile stimulation of the rubber hand and participants' own hand. We measured body image representation by means of weight estimation errors during the SWI. Measures of eating disorder symptomatology
(Eating Disorders Examination Questionnaire [EDE-Q]; Fairburn \& Beglin, 1994, 2008) and self-objectification (Self-Objectification Questionnaire; Fredrickson, Roberts, Noll, Quinn, \& Twenge, 1998) were also included to control for their potential role in multisensory integration.

\section{METHODS \\ Participants}

Forty-one heterosexual female participants were recruited through the University College London research participation system. They were aged between 18 and 40 years ( $M=25.13$ years, $S D=4.21$ years). Participants were not taking any medication (including the contraceptive pill) and were recruited in the follicular phase of their menstrual cycle (between the fifth and 14th day) to control for hormonal levels (Salonia et al., 2005). Exclusion criteria included being left-handed, pregnant, or currently breastfeeding (see MacDonald et al., 2011); a history of any medical, neurological, or psychiatric illness; BMI out of the range $18.5-24.9(M=21.38, S D=2.64)$; use of any illegal drugs within the last 6 months; and consumption of more than five cigarettes per day. Participants were asked to refrain from consuming any alcohol the day before testing and any alcohol or coffee on the day of testing. All participants provided informed consent to take part and received a compensation of $\$ 40$ for travel expenses and time. Ethics approval was obtained by University College London, and the study was carried out in accordance with the provisions of the Declaration of Helsinki 1975, as revised in 2008. One participant was excluded because she discontinued the study (i.e., she took part in only one of two testing sessions); four participants were later excluded from the analysis as they were found to be extreme outliers in their embodiment scores in the placebo condition (embodiment score $\pm 3 S D$ from group mean). The final sample was composed of 36 participants $\left(M_{\text {age }}=25.03\right.$ years, $S D=3.96$ years $)$.

\section{Experimental Design}

The study employed a double-blind AB/BA (oxytocinplacebo/placebo-oxytocin) crossover design, with compound (IN-OT vs. placebo) as the within-participant factor. Each participant participated in two identical sessions, each lasting between 1.5 and $2 \mathrm{hr}$ and conducted between 1 and 3 days apart; this was to ensure that they were tested in the same phase of the menstrual cycle. The testing sessions always took place between 9:00 a.m. and 12:00 p.m.. In one session, participants were asked to self-administer 40 IU of oxytocin; and in the other session, placebo (see Figure 1) in a counterbalanced and double-blinded manner. Participants were randomly allocated to the nasal spray sequence (AB/BA); 18 participants received placebo on the first visit and IN-OT on the second visit, and another 18 received the reverse order 
Figure 1. Study design and flowchart.

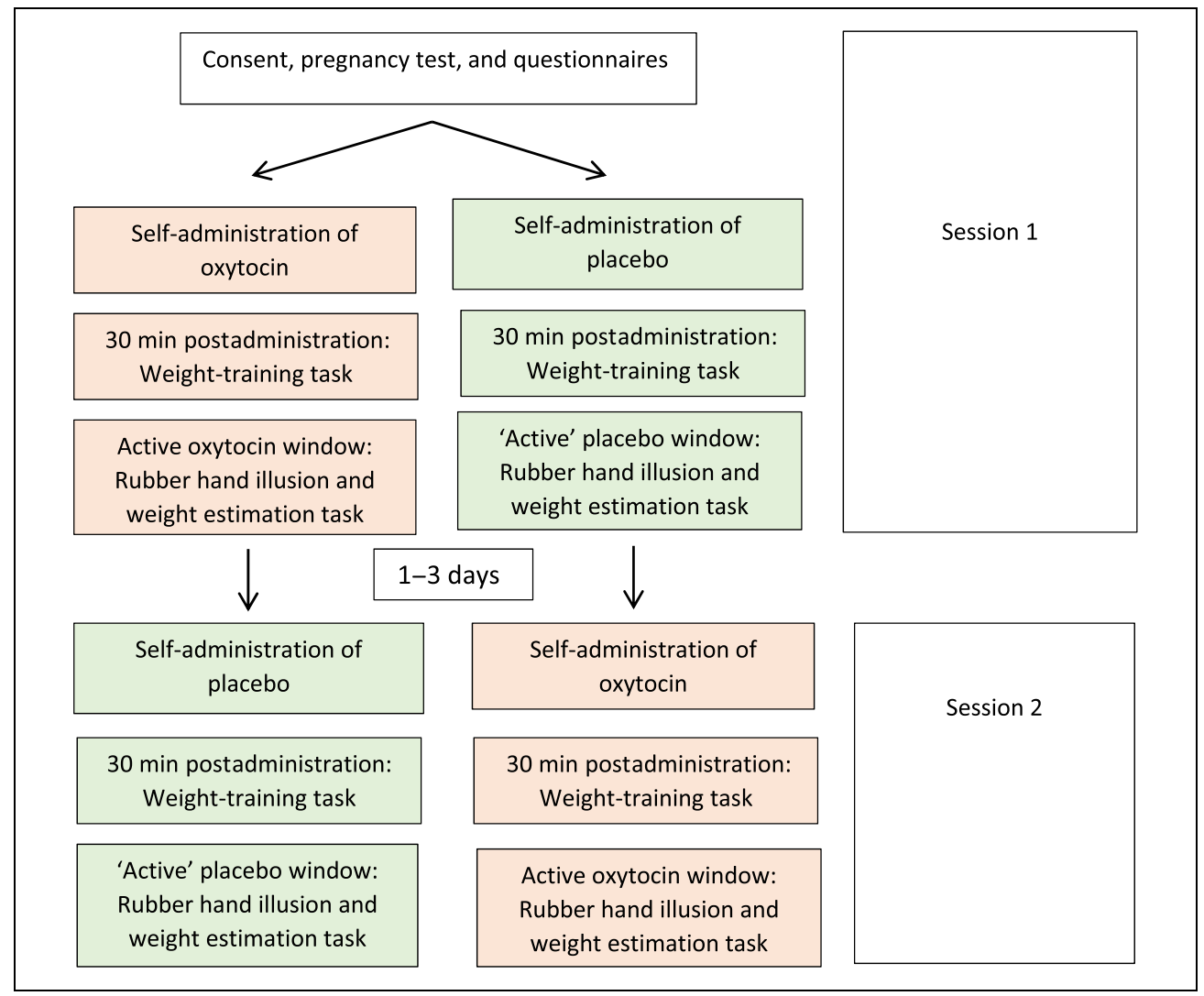

(IN-OT on the first visit and placebo on the second visit). The order of administration was included as a covariate in all analyses. The RHI and SWI (see below for details of procedure) were administered $30 \mathrm{~min}$ after nasal spray administration (see Paloyelis, Doyle, et al., 2016; MacDonald et al., 2011, for optimal temporal window). The asynchronous condition of the RHI was run only once to establish the presence of the illusion, whereas the synchronous condition was repeated twice, once with a "normal"-sized hand and once with a "larger" hand (see below for more details), to investigate the effect of hand size on the embodiment process. The order of the three conditions (normal hand/synchronous, normal hand/asynchronous, and larger hand/synchronous) was pseudorandomized between participants (i.e., such that the larger hand was never administrated as the first condition so as to always obtain the first visual capture measure with the normalsized hand), but it was kept constant within participants between the two testing sessions.

\section{Oxytocin and Placebo Spray}

Participants received 40 IU of oxytocin (Syntocinon, Novartis Pharmaceutical) or placebo (containing the same ingredients as Syntocinon except the active ingredient) by means of a nasal spray. Two practice bottles containing water were used for the participants to familiarize themselves with the procedure, one for the experimenter to demonstrate the correct technique and one for the participant to practice. Experimental instruction about the nasal spray administration procedure, the position of the head and of the nasal spray inside the nose, and breathing technique were given to the participants. Before the beginning of the self-administration procedure, all the participants were asked to blow their nose. Participants self-administered a puff containing 4 IU every $30 \mathrm{sec}$ alternating between nostrils (five for each nostril), for a total of 10 puffs. Half of the sample started the administration on the right nostril; and half, on the left nostril. Participants were specifically instructed to not blow their nose during the administration procedure. At the end of the last puff of nasal spray, participants were given 3 min of resting time in which they were instructed to relax. The self-administration procedure took approximately $9 \mathrm{~min}$, including $3 \mathrm{~min}$ of rest at the end (see also Paloyelis, Krahé, et al., 2016).

\section{RHI}

\section{Procedure}

The RHI was performed following the procedure fully described in previous studies (Crucianelli, Krahé, Jenkinson, \& Fotopoulou, 2018; Crucianelli, Metcalf, Fotopoulou, \& Jenkinson, 2013). Two adjacent stroking areas, each measuring $9 \mathrm{~cm}$ long $\times 4 \mathrm{~cm}$ wide, were identified and marked with a washable marker on the hairy skin of 
participants' left forearm (wrist crease to elbow; McGlone et al., 2012). Tactile stimulation (i.e., stroking) was alternated between these two areas to minimize habituation, and congruent stroking area changes were applied to the rubber hand in all instances (Crucianelli et al., 2013). In each condition, the experimenter placed the participants' left hand (palm facing down; fingers pointing forward) at a fixed point inside a wooden box $(34 \mathrm{~cm} \times$ $65 \mathrm{~cm} \times 44 \mathrm{~cm})$.

A prestroking estimate of finger position was then obtained (for the measurement of proprioceptive drift) using a tailor's tape measure placed on top of the box lid. Subsequently, the rubber arm was positioned in the right half of the box (participant-centered reference frame), in front of the participant's body midline, and in the same direction as the participant's actual left arm. The distance between the participant's left arm and the visible arm (on the sagittal plane) was approximately $28 \mathrm{~cm}$. A wooden lid prevented visual feedback of the participant's own arm. The participant also wore a black cape to occlude vision of the proximal end of the rubber arm and the participant's left arm. The rubber hands were custom made by an artist with expertise in body-part modeling, by means of hand casts obtained from two volunteers, one with a BMI within the healthy weight range (healthy BMI = 18.5-24.9) and one having a $\mathrm{BMI}$ in the obese range (obese BMI = 30-39.9). Each cast was subsequently hand painted to create a life-like model hand (Figure 2).

Participants were then asked to look at the rubber hand continuously for $15 \mathrm{sec}$, before completing the preembodiment questionnaire (i.e., visual capture effect). Tactile stimulation was then applied for 1 min using two identical, cosmetic makeup brushes (Natural Hair Blush Brush, $\mathrm{N}^{\circ} 7$, The Boots Company), using the speed of $3 \mathrm{~cm} / \mathrm{sec}$ (Crucianelli et al., 2013). In the synchronous conditions, the participants' left forearm and the rubber forearm were stroked such that visual and tactile feedback were congruent, whereas in the asynchronous conditions, visual and tactile stimulation were temporally incongruent (i.e., offset by $2 \mathrm{sec}$ ). After the stimulation period, the felt and actual location of the participants' left index finger was again measured following the preinduction procedure. After the tactile stimulation period, participants completed the poststroking embodiment questionnaire. Before commencing the next condition, they were given a 60 -sec rest period, during which they were instructed to freely move their left hand.

\section{Outcome Measures}

After positioning the hand inside the box, participants were asked to close their eyes and to indicate on the ruler with their right hand the position where they felt that their own left index finger was inside the box. The experimenter then measured and recorded the actual position of the participants' left index finger. After the stimulation period, the felt and actual location of the participants' left index finger was again measured following the preinduction procedure. The difference between the preerror and posterror in location represents a measure of proprioceptive drift.

An embodiment questionnaire (Longo, Schüür, Kammers, Tsakiris, \& Haggard, 2008) was used to capture the subjective experience of the illusion (12 statements rated on a 7-point Likert-type scale; $-3=$ strongly disagree, $+3=$ strongly agree). In each condition, the questionnaire was administered prestroking (i.e., embodiment due to the visual capture effect) and poststroking, and we calculated their difference to obtain a measure of subjective embodiment due to visuotactile integration (i.e., change in embodiment, as in Crucianelli et al., 2013, 2018). This questionnaire consists of three subcomponents: "felt ownership," which is related to the feeling that the rubber hand is part of one's body; "felt location" of own hand, which is related to the feeling that the rubber hand and one's own hand are in the same place; and affect, which includes items related to the
Figure 2. The SWI procedure. First, participants completed an RHI procedure where they were asked to look at a regular-sized rubber hand (on the right) or a larger rubber hand (on the left). Participants were asked to estimate the weight of three cans, each having an identical size but different weights (i.e., 125,150 , and $175 \mathrm{~g}$ ). The experimenter passed the can to the participant through the hole in the box. Participants were instructed to lift the can, without shaking it, and to give their best estimation about its weight. No feedback was provided to the participants.

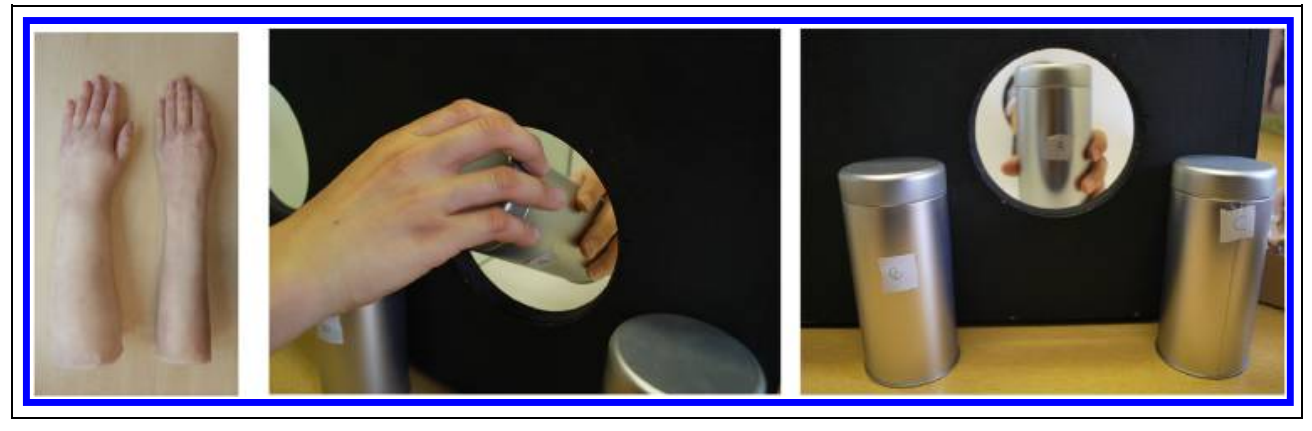


experience being interesting. We examined this difference between prestroking and poststroking (change in embodiment) for each of the statements separately as well as for an overall "embodiment of rubber hand" (Longo et al., 2008) score, which was obtained by averaging the scores of the two subcomponents specifically related to embodiment, namely, ownership and felt location, that did not relate to affect. The affect subcomponent also included a measurement of the perceived pleasantness of the tactile stimulation quantified by means of a visual analog scale ranging from 0 (not at all pleasant) to 100 (extremely pleasant).

\section{SWI \\ Procedure}

The SWI task was based on the procedures described by Haggard and Jundi (2009). Before the main experiment (see timeline in Figure 1), participants received training in weight estimation. They were asked to lift two opaque cylinders (i.e., metal tea caddies/containers; Figure 2) that contained wooden beads. Each cylinder was of the same size (height $=15.5$, diameter $=\sim 7.5 \mathrm{~cm}$ ) but varied in weight (either 100 or $200 \mathrm{~g}$ ). These same cylinders were later used in the actual experimental task. Participants were asked to estimate the weight of the cylinders 10 times by lifting them with their left hand and putting them back down: five times they were given the cylinder weighing $100 \mathrm{~g}$ and five times they were given the cylinder weighing $200 \mathrm{~g}$, in a random order. During this training stage, participants were given feedback about their performance (i.e., if they were right or wrong) and were informed about the actual weight of the cylinder after each trial. By the end of the training session, all participants successfully learnt to distinguish between cylinders weighing 100 and 200 g (i.e., the cutoff was 8 out of 10 correct trials in a row). This training allowed participants the opportunity to familiarize themselves with the experimental stimuli and ensured that all participants started the main experiment with the same weight reference points (see also Haggard \& Jundi, 2009).

Subsequently, the baseline weight estimation took place. The weight estimation task consisted on lifting three cans of the same shape and size (described above) but different weights $(125,150$, or $175 \mathrm{~g}$, presented in a random order across conditions and participants). The experimenter placed the can between the participants' left index finger and thumb. Participants were asked to gently lift the can without shaking it, to put it down, and to provide verbally the best estimation about its weight (to the nearest gram). Instructions included the information that the weight could be anything between 100 and 200 g but not exactly 100 or 200 g; no feedback about the estimation was given at this stage. During the session, participants completed the weight estimation task twice more: following the RHI with the normal-sized hand and following the RHI with the larger hand. The difference between the estimated and actual weight was the measure of the SWI (i.e., weight estimation error), which was then compared between the conditions after embodiment of the normal-sized or larger hand to investigate the relationship between expectations driven by the illusory body size perception and the estimated weight of the objects.

\section{SOQ}

The SOQ (Fredrickson et al., 1998) measures the extent to which individuals see their bodies in observable, appearance-based (i.e., objectified) terms, versus nonobservable competence-based terms. The questionnaire consists of 10 body attributes (e.g., attractiveness, strength, health), which participants are required to rank by how important each is to their own physical self-concept, from 0 (least impact) to 9 (greatest impact). Self-objectification scores are calculated by subtracting the summed ranks given to the five competence-based attributes (e.g., health, energy) from the summed ranks of the five appearancebased attributes (e.g., physical attractiveness, body measurements). Scores range from 25 to 225 , with higher scores indicating a greater emphasis on appearance, which is interpreted as greater self-objectification. The SOQ has good test-retest reliability $(r=.92$, cited in Miner-Rubino, Twenge, \& Fredrickson, 2002).

\section{$E D E-Q$}

The EDE-Q 6.0 (Fairburn \& Beglin, 1994, 2008), which has good consistency and reliability (global score $\alpha=.90$; Peterson et al., 2007), was used to measure eating disorder symptomatology. The questionnaire consists of 28 items rated on a 7-point Likert scale (ranging from no days/not at all to every day/markedly) and six items asking about frequency of behavior. The questionnaire can be divided into four subscales ("dietary restraint," e.g., Have you been deliberately trying to limit the amount of food you eat to influence your shape or weight?; "eating concern," e.g., Over the past 28 days, how concerned have you been about other people seeing you eat?; "weight concern," e.g., Has your weight influenced how you think about yourself as a person?; "shape concern," e.g., Have you had a definite desire to have a totally flat stomach?) or a single global measure. To obtain an overall or "global" score, the four subscale scores are summed and the resulting total is divided by the number of subscales (i.e., four). The clinical cutoff is 2.8 on the global score (Mond et al., 2008).

\section{General Procedure}

The experiment was run by two female experimenters. Upon arrival, in the first session only, participants were asked to provide a urine sample for a pregnancy test (pregnancy test device, SureScreen Diagnostics), which 
was carried out to exclude the possibility of any ongoing, unknown pregnancy that might be adversely affected by the administration of IN-OT. After confirmation of the negative result of the pregnancy test, participants completed the EDE-Q and the SOQ (presented in a random order), and their arm was prepared for later administration of the RHI (see RHI section above). Subsequently, participants self-administered, under both experimenters' supervision, either IN-OT or the placebo. The order of the treatment was counterbalanced across participants, and both experimenters and participants were blind to the treatment order (as detailed in Oxytocin and Placebo Spray section above).

During the 30 min after spray administration (see Paloyelis, Doyle, et al., 2016; MacDonald et al., 2011, for optimal temporal window), no social contact between the participant and the experimenters took place beyond necessary experimental instructions. Participants were asked to refrain from checking their phones or doing any personal reading. During this waiting time of oxytocin activation, participants were familiarized with the weight estimation task, before completing the three weight estimation baselines (described in SWI section above). In the remaining time, participants were offered the opportunity to complete a sudoku or word search puzzle. At the beginning of the active oxytocin window, participants completed the RHI and the SWI for the following $40 \mathrm{~min}$ (Figure 3). Participants were fully debriefed and reimbursed $\$ 40$ for their time at the end of the second study visit.

\section{Statistical Analysis}

This study aimed to explore whether IN-OT versus placebo would affect (1) the subjective feeling of ownership toward the rubber hand, as measured using an embodiment questionnaire; (2) proprioceptive judgments regarding the location of the position of the real hand relative to the rubber hand (proprioceptive drift); and (3) the representation of body size, as measured by means of an SWI, quantified as weight estimation error after the embodiment of rubber hands of different sizes.

The multivariable analyses were performed using a purposeful selection of covariates (Hosmer, Lemeshow, \& May, 2008). After this procedure, preliminary correlational analyses were conducted to investigate the relationships between the psychometric measures, namely, the SOQ and the EDE-Q, with the subjective measure of the RHI, the proprioceptive drift, and the weight estimation error (see Table 1 ). In case of a $p$ value $<.20$, we included these variables as covariates in the analyses (Hosmer et al., 2008). According to this criterion, the EDE-Q correlated with the subjective embodiment in the synchronous condition after placebo $(p=.08)$ and with the weight estimation error after embodiment of the larger hand in the placebo condition only $(p=$ .12). After running these two linear mixed models (LMMs), we planned to follow up these with another two LMM analyses, which included only the variables that significantly contributed to the model at a $p$ value $<.05$, to specify the contribution of these variables only to the final model (Hosmer et al., 2008). The EDE-Q did not meet this criterion in the first and second LMMs ( $p s=$ .64 and .84 , respectively), and therefore it was not included in the final model. The main analyses were conducted by means of LMM analyses that allow the use of both fixed and random effects in the same analysis. Specifically, the effect of IN-OT versus placebo on the RHI was analyzed by means of three separate LMM analyses. One analysis was run to test the effect of IN-OT versus placebo on the visual capture of hands of different

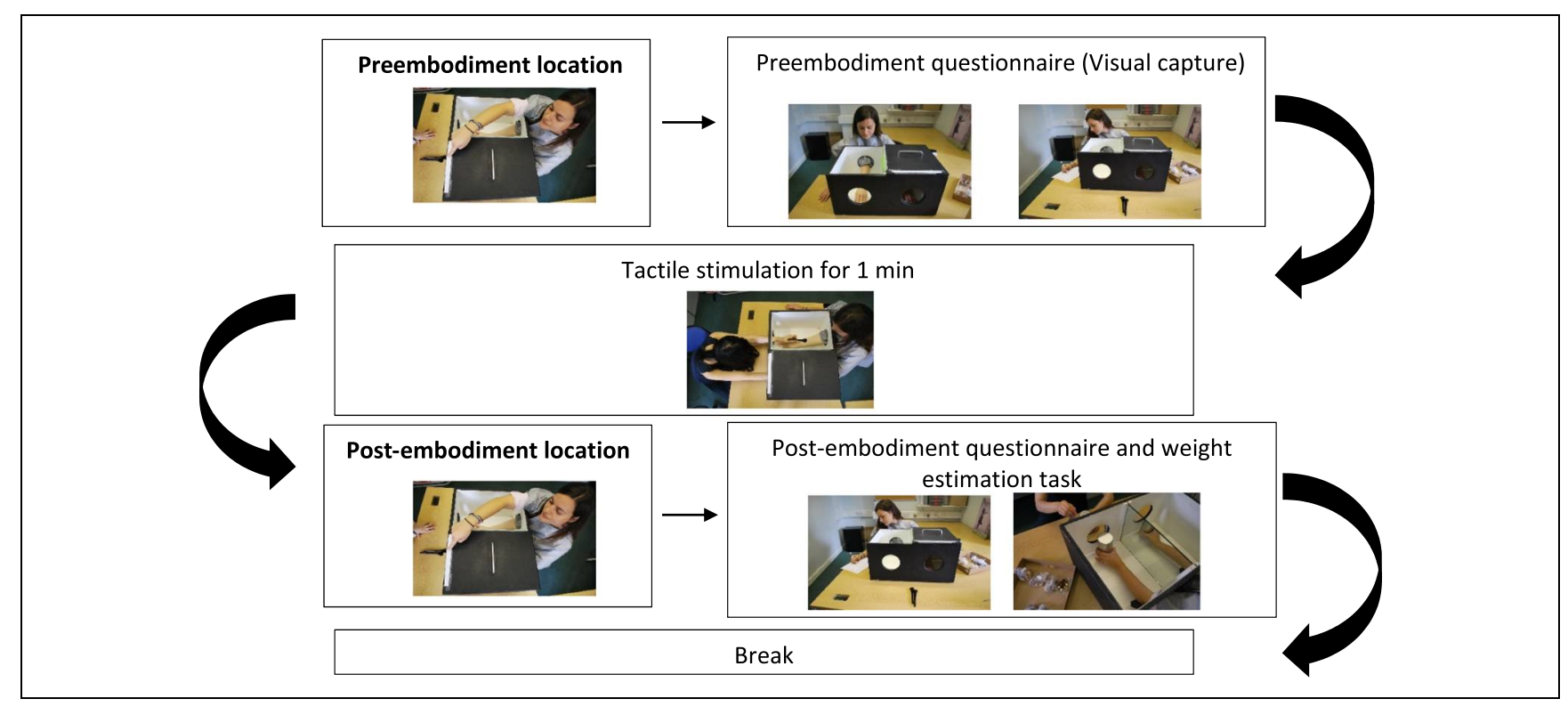

Figure 3. The RHI and SWI procedure. 
Table 1. Pearson's Correlational Analyses between SOQ or EDE-Q and Measures of Embodiment

\begin{tabular}{cc}
\hline SOQ & $E D E-Q$ \\
\hline Classic RHI: subjective change in embodiment &
\end{tabular}

Classic RHI: subjective change in embodiment

IN-OT synchronous touch

$\begin{array}{rrr}r & -.17 & -.19 \\ p & .29 & .24\end{array}$

IN-OT asynchronous touch

$r$

$p$
$-.00$

.98

PL synchronous touch

$\begin{array}{rrr}r & -.06 & -.28 \\ p & .71 & .08\end{array}$

PL asynchronous touch

$$
r
$$$$
p
$$

Classic RHI: proprioceptive drift

IN-OT synchronous touch

$\begin{array}{lr}r & .04 \\ p & .80\end{array}$

IN-OT asynchronous touch

$r$

$p$

$-.06$

.71
PL synchronous touch

$r \quad .06$
$p$

$p \quad .74$

PL asynchronous touch

$r \quad 10$

\begin{tabular}{l}
$p \quad .55$ \\
\hline
\end{tabular}

SWI: error in weight estimation

IN-OT regular hand

$\begin{array}{rrr}r & .02 & -.07 \\ p & .88 & .67\end{array}$

PL regular hand

$\begin{array}{lr}r & -.01 \\ p & .97\end{array}$

IN-OT larger hand

\begin{tabular}{lrr}
$r$ & .11 & -.08 \\
$p$ & .52 & .64 \\
\hline
\end{tabular}

Table 1. (continued)

\begin{tabular}{lcc}
\hline & $S O Q$ & $E D E-Q$ \\
\hline PL larger hand & \\
$r$ & .04 & .25 \\
$p$ & .80 & .12 \\
\hline
\end{tabular}

The values reported are the correlational coefficients $r$ in the first row and the $p$ values in the second row.

sizes (i.e., comparison between normal-sized and larger hands on the preembodiment questionnaire only and the interaction between hand size and oxytocin on measures of embodiment). The second LMM analysis was run to test the effect of IN-OT versus placebo on the occurrence of the illusion (i.e., comparison between synchronous and asynchronous touch condition, and the interaction between synchronicity and oxytocin, on measures of embodiment). The final LMM analysis was run to test the effect of IN-OT versus placebo on the hand size manipulation (i.e., comparison between normal-sized and larger hands in synchronous conditions only, and the interaction between hand size and oxytocin, on measures of embodiment). Finally, the latter analysis was repeated with the weight estimation as the dependent variable, to investigate the effect of IN-OT versus placebo on the occurrence of the SWI.

In all these analyses, order of compound administration (oxytocin-placebo or placebo-oxytocin) was included as a covariate. The baseline weight estimation was included in all the analyses of the SWI effect as a covariate. All data were analyzed using SPSS, Version 23.

\section{RESULTS}

\section{Preliminary Analyses}

Preliminary correlational analyses showed no significant relationships between self-objectification (SOQ) or eating disorder symptomatology (EDE-Q) with the subjective measure of the RHI (embodiment questionnaire), proprioceptive drift, or the weight estimation error in the SWI. Results are reported on Table 1. Given the lack of significant relationships between the psychometric measures and the main measures of interest, SOQ and EDE-Q were not taken into account in subsequent analyses.

\section{IN-OT Effects on Visual Capture of Hands of Different Sizes as Measured by Self-report}

To assess whether IN-OT affects the visual capture of hands of different sizes during the RHI, an LMM analysis was run to explore the effect of IN-OT (vs. placebo) and watching a larger versus regular-sized hand on the embodiment resulting from visual capture only. Order of nasal spray administration was considered as a covariate. This analysis showed that neither hand size 
$(F(1,35)=1.69, p=.20, r=0.46)$ nor oxytocin $(F(1$, $35)=0.74, p=.39, r=0.09)$ has a significant main effect on the visual capture. The interaction between nasal spray and hand size was nonsignificant $(F(1,35)=1.28, p=$ $.27, r=.27)$.

\section{IN-OT Effects on Body Ownership: Synchronous vs. Asynchronous Stimulation}

Change in subjective embodiment as measured by selfreport To assess whether IN-OT affects the subjective reporting of body ownership during the RHI, an LMM analysis was run to explore the effect of IN-OT (vs. placebo) and synchronous (vs. asynchronous) stimulation on the change in embodiment questionnaire scores. The order of administration was considered in the analysis as a covariate. This analysis revealed a main effect of synchronicity $(F(1,35)=8.8, p=.005, r=.82$, see Figure 4$)$, with synchronous touch $(M=0.75, S E=0.16)$ leading to greater embodiment compared with asynchronous touch $(M=-0.01, S E=0.18)$. This result confirmed the occurrence of the illusion from a subjective point of view. Nasal spray did not have a significant main effect on the change in embodiment $(F(1,35)=0.48, p=.49)$. However, there was a significant interaction between synchronicity and nasal spray $(F(1,35)=4.87, p=.034$, $r=.64)$. Bonferroni-corrected post hoc analyses (adjusted $\alpha=.025$ ) revealed that IN-OT (compared with placebo) increased embodiment of the rubber hand in the synchronous $(t(35)=12.50, p=.001, r=.90)$ but not in the asynchronous $(t(35)=0.05, p=.82, r=.01)$ condition.

Embodiment changes as measured by proprioceptive drift To assess whether IN-OT affects the perceived location of the hand in the RHI, an LMM analysis was run to explore the effect of IN-OT (vs. placebo) and synchronous (vs. asynchronous) stimulation on the proprioceptive drift. The order of administration was considered in the analysis as a covariate. This analysis revealed no significant main effect of synchronicity $(F(1,35)=0.64$, $p=.43, r=.12)$ and IN-OT $(F(1,35)=0.29, p=.59, r=$
.04) nor a significant interaction between synchronicity and nasal spray $(F(1,35)=0.51, p=.48, r=.08)$. Given the lack of findings regarding proprioceptive drift on our main RHI induction, we did not conduct further analyses on proprioceptive drift.

\section{IN-OT Effects on Embodiment of Hands of Different Sizes as Measured by Self-report}

To assess whether IN-OT affects the embodiment of hands of different sizes during the RHI, an LMM analysis was run to explore the effect of IN-OT (vs. placebo) and watching a larger versus regular-sized hand on the subjective change in embodiment. Order of nasal spray administration was considered as a covariate. This analysis showed a main effect of oxytocin $(F(1,35)=$ $10.51, p=.003, r=.87)$, with oxytocin leading to a greater embodiment (embodiment oxytocin: $M=0.81$, $S E=0.13$ ) compared with placebo (embodiment placebo: $M=0.74, S E=0.14)$. This result is in line with the one reported above; that is, IN-OT seems to enhance the embodiment of the rubber hand more than placebo regardless of the size of the hand, when touch is synchronous (i.e., no asynchronous condition was conducted with the larger hand). Indeed, hand size did not have a significant main effect on the change in embodiment $(F(1,35)=3.43, p=.07, r=.25)$. The interaction between nasal spray and hand size was nonsignificant $(F(1,35)=0.09, p=.77, r=.02)$. In summary, (1) we found that IN-OT compared with placebo enhanced the embodiment of the rubber hand, (2) we did not find an effect of hand size on embodiment of the rubber hand, and (3) we did not find that IN-OT enhances the RHI depending on rubber hand size.

\section{IN-OT Effects on Change in Body Size Representation: The SWI}

Finally, an LMM analysis was run to assess the effect of nasal spray and hand size on the weight estimation task, quantified as weight estimation error. The order of administration was considered in the analysis as a covariate,
Figure 4. (A) Mean of raw embodiment scores (mean corrected) for the synchronous and asynchronous stroking conditions, after administration of oxytocin or placebo. (B) Mean of raw weight estimation error (mean corrected) after embodiment of the regularsized and larger rubber hands, after administration of oxytocin or placebo. Error bars denote standard error. ${ }^{*} p<.05$.

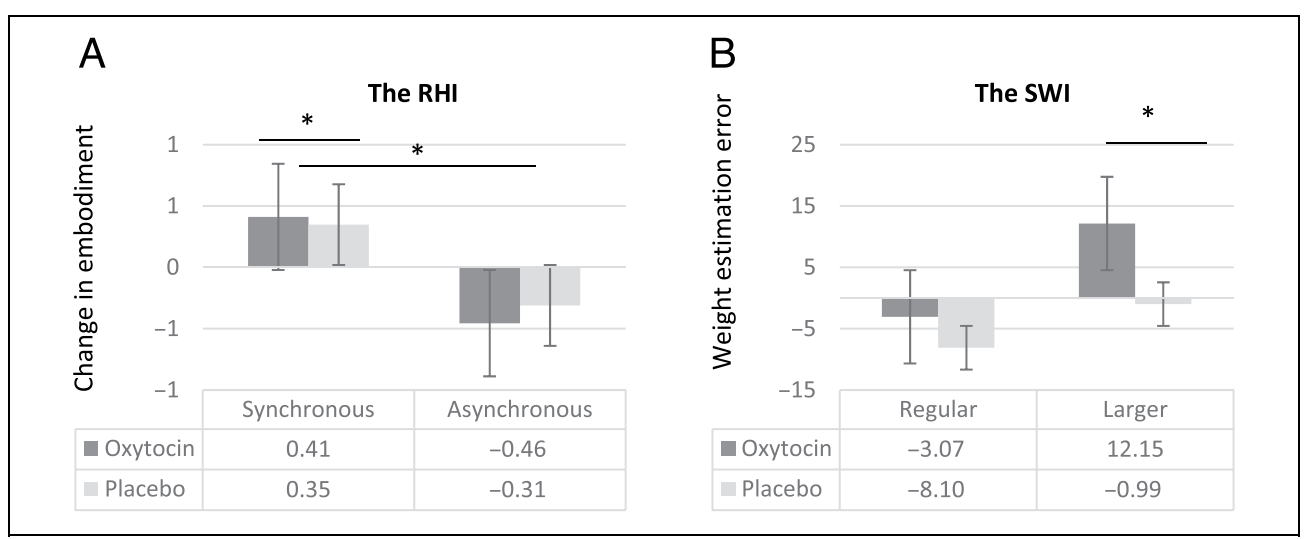


together with the baseline weight estimation. This analysis showed that neither hand size $(F(1,35)=0.04, p=$ $.95, r=.01)$ nor nasal spray $(F(1,35)=1.89, p=.18, r=$ .30) had a significant main effect on the weight estimation task. However, there was a significant interaction between hand size and nasal spray $(F(1,35)=7.2, p=$ $.01, r=.77$; see Figure 3). Bonferroni-corrected post hoc analyses $(p=.025)$ showed no effect of oxytocin compared with placebo in weight estimation error for the regular hand $(t(35)=-0.67, p=.51, r=.19)$. By contrast, oxytocin increases the weight estimation error after embodiment of the larger hand, compared with placebo $(F(35)=-1.87, p=.007, r=.30)$. These results indicate that IN-OT enhances the SWI in comparison with placebo when a larger hand is used, but not when a regular-sized hand is used.

\section{DISCUSSION}

This double-blind, placebo-controlled, crossover study aimed to explore the effect of IN-OT, in comparison with placebo, on multisensory integration in relation to the sense of body ownership (i.e., RHI) and body image (i.e., SWI). Specifically, we investigated whether IN-OT versus placebo would affect (1) the subjective feeling of body ownership, as measured by means of the embodiment questionnaire; (2) the capture of proprioception by touch, as measured by means of proprioceptive drift; and (3) the representation of body size, as measured by means of weight estimation error after embodying rubber hands of different sizes (i.e., SWI).

The results showed that IN-OT enhances the subjective feeling of ownership toward a rubber hand to a greater extent compared with placebo. This effect is independent of the size of the seen hand. In fact, participants embodied the large hand to the same extent as the regular-sized hand, and IN-OT increases the feeling of embodiment regardless of the hand's size. In contrast, IN-OT did not affect the proprioceptive drift differently compared with placebo. Finally, this study showed that IN-OT enhanced the SWI after embodiment of a large hand only, in the sense that, after administration of IN-OT, participants tend to overestimate the weight of the cans to a greater extent compared with the placebo control condition. Such effect was not present after embodiment of the regular-sized hand.

Our findings confirmed our first hypothesis that embodiment of the rubber hand would be enhanced after administration of IN-OT only. Specifically, on the basis of theoretical proposals about the role of oxytocin in somatosensory attenuation (Quattrocki \& Friston, 2014), we hypothesized that IN-OT would enhance the RHI effects, by attenuating the precision of epistemically private somatosensory signals relative to the precision of visual signals, such as the vicarious touch on the rubber hand. Our results showed that IN-OT modulated multisensory integration and corresponding subjective measure of body ownership (i.e., embodiment questionnaire) over the RHI only during synchronous tactile stimulation of the rubber hand and participants' own hand. No effect of IN-OT was observed in the behavioral (i.e., proprioceptive drift) measure of body ownership over the RHI, nor in the mere visual capture conditions. These findings seem to suggest that IN-OT affects the multisensory integration processes only when synchronous tactile stimulation is involved, but not in the condition when only visual and proprioceptive signals need to be integrated (i.e., IN-OT does not enhance the visual capture of ownership seen in previous studies; see Martinaud et al., 2017; Samad et al., 2015; Pavani et al., 2000). In terms of the predictive coding hypothesis we have put forward, this might suggest that oxytocin, as a neuromodulator, plays a role in precision weighting (i.e., increasing precision) of conflicting sensations deriving from the rubber hand (vicarious touch) in contrast with the ones from the self (epistemically private touch). This process reflects subjective feelings of body ownership toward the rubber hand, but not in an update in the perceived location of the hand (see Abdulkarim \& Ehrsson, 2016; Rohde, Di Luca, \& Ernst, 2011, for experimental evidence on the dissociation between these two measures of the RHI). In addition, the lack of significant findings in the context of visual capture might suggest that IN-OT does not modulate precision weighting of conflicting proprioceptive sensations deriving from the rubber hand (i.e., where I see the hand to be) and from the participant's own hand (i.e., where I feel the hand to be). This might provide further support to our hypothesis that IN-OT modulates the precision of sensorimotor, tactile signals.

To our knowledge, only one recent study attempted to explore the relation between oxytocin and the sense of body ownership. Ide and Wada (2017) showed that the subjective, but not objective, experience of the RHI is associated with oxytocin, in the sense that participants with a higher level of salivary oxytocin at baseline showed an increased experience of the RHI. The authors speculated that oxytocin might enhance the precision of tactile stimulation by modulating the activity of the insular cortex, and the higher susceptibility to the experience of the RHI would be a consequence of an increase in resources (i.e., attention) placed on touch (Ide \& Wada, 2017). This interpretation is in line with our hypothesis that IN-OT might increase the precision of vicarious touch on the rubber hand. However, here, we distinguish between vicarious (seen) touch and epistemically private (felt) touch, and we also propose that IN-OT might reduce the precision of the latter relative to visual signals. Moreover, we note that the instability of single measurements of oxytocin in peripheral fluids (Amico, Tenicela, Johnston, \& Robinson, 1983), the correlational nature of the findings, the unclear relationship between peripheral oxytocin levels and central oxytocin (Kagerbauer et al., 2013; Ludwig \& Leng, 2006), and the small sample size $(n=15)$ of Ide and Wada's study should warrant 
caution against premature conclusions relating to their findings.

The present data also partially support our second hypothesis that hand size was not expected to have an effect on the RHI per se (e.g., Farmer, TajaduraJiménez, \& Tsakiris, 2012; Haggard \& Jundi, 2009) but on the weight estimation error, here included as an indirect measure of body image (Haggard \& Jundi, 2009). Indeed, the sense of body ownership (here manipulated by means of the RHI) can be measured by means of an explicit embodiment questionnaire. In contrast, the SWI has been included here as a more implicit measure of body image. We hypothesized that the effect of hand size would be stronger for the implicit compared with the explicit measure of body representation, suggesting a dissociation between explicit and implicit facets of body representation. Our findings are in line with previous studies showing that the RHI is object dependent, in the sense that it occurs as long as the object could be a body part (Tsakiris, 2010). In addition, the physical characteristics of the hand, such as skin tone (Farmer et al., 2012), do not seem to affect the occurrence of the illusion. Similarly, our findings showed that the size of the hand did not block or reduce the embodiment process toward the rubber hand. In other words, the effect of IN-OT seems to be over and above the physical characteristics of the hand, and it is rather dependent on the synchronicity between seen and felt touch. Furthermore, we predicted that IN-OT would modulate multisensory integration and corresponding behavioral measure as quantified by judgments of the weight of external objects having a constant size but different weights after embodiment of the larger hand only (as in Haggard \& Jundi, 2009). In other words, we expected IN-OT to increase the error in weight estimation after the embodiment of a large hand only; in contrast, no effect of IN-OT on weight estimation would be observed after the embodiment of the regular-sized hand. Participants showed a greater weight estimation error after the embodiment of the larger hand as compared with the regular-sized hand (i.e., main effect of hand size on weight estimation error); however, this effect did not reach significance (see Haggard \& Jundi, 2009). Nevertheless, the results confirmed our hypothesis by showing a significant interaction between hand size and nasal spray. IN-OT seems to promote the occurrence of the SWI compared with placebo, in the sense that it increases the weight estimation error only after embodiment of the larger hand. These findings might suggest that IN-OT allows more flexibility in terms of body shape and size. We speculate that this process might be related to the role that oxytocin plays in pregnancy, a moment of important bodily and emotional changes in women.

In addition, the fact that IN-OT influences explicit and implicit measures of the malleability of body representation is in line with previous findings showing that oxytocin sharpens the recognition of basic emotions as well as hidden/implicit emotional facial expressions (Leppanen, Ng, Tchanturia, \& Treasure, 2017; Leknes et al., 2013, for a meta-analysis). This explanation supports the idea that oxytocin might play an important evolutionary role, by promoting social affiliation and bonding and by downregulating pain perception associated with reproduction (Paloyelis, Krahé, et al. 2016; Insel, 1992).

In conclusion, our findings are the first to suggest that IN-OT modulates processes of multisensory integration in relation to both body ownership and body image. We speculate that this process might follow basic principles of predictive coding. However, the interpretation we put forward in this article is tentative, and we hope it will pave the way for future studies that might investigate the relationship between oxytocin and somatosensory precision more specifically. In particular, studies using computational modeling approaches (e.g., Samad et al., 2015) or measuring synaptic gain (Zeller et al., 2014, 2016) during the RHI with modulation of oxytocin could test our hypotheses and provide unique insight into the mechanisms by which oxytocin may act to modulate the subjective feelings of body ownership, as we speculate in this article. In the context of multisensory integration, oxytocin seems to mediate the precision weighting (or attention) given to descending prior prediction and ascending sensory inputs (prediction errors). When a hand is placed in front of the participant, in peripersonal space and from a first-person perspective, the participant has a strong prediction that the hand he or she sees must belong to him or her. When there are "incongruent" proprioceptive (i.e., where I see the rubber hand to be vs. where I feel my own hand to be) and visuotactile (i.e., vicarious touch on the RHI vs. felt touch on my own hand) signals, then the brain must resolve this conflict by downregulating incongruent or incompatible sources of information. In this context, we hypothesized that IN-OT might attenuate epistemically private sensations in social situations, to allow us to build a self during social interactions characterized by some degree of "interpersonal synchrony" (Fotopoulou \& Tsakiris, 2017). Hence, the self is built by "identification" (see Panagiotopoulou et al., 2017), in this context meaning the integration of epistemically private sensations and exteroceptively perceived but "synchronous" sensations from others. We proposed that IN-OT might enhance this process of identification by increasing the feeling that our body feels like our own, even when we perceive it via exteroception. In other words, IN-OT might mediate the neurobiological mechanism promoting and sharpening the precision of exteroceptive sensory inputs (e.g., visual signals of a realistic hand in peripersonal space and of the seen touch) to determine the weighting of top-down cognitive predictions against bottom-up, epistemically private sensory information (e.g., felt location and touch), ultimately facilitating multisensory integration.

Furthermore, IN-OT may modulate the sense of body ownership by means of other possible mechanisms. For 
example, according to Quattrocki and Friston (2014), oxytocin might act by attenuating interoceptive prediction errors, ultimately increasing the relative precision of exteroceptive information. This interpretation could be partially supported by studies showing physiological changes to the participants' real hand (e.g., a reduction in skin temperature) during the RHI, which are consistent with the idea that interoceptive prediction errors might be attenuated (Moseley et al., 2008). However, recent studies have cast doubt on the replicability of these physiological findings in the context of the RHI, and therefore this hypothesis should be interpreted with caution (e.g., Crucianelli et al., 2018; de Haan et al., 2017).

To our knowledge, this is the first study to have investigated whether the oxytocinergic system might modulate process of multisensory integration via embodied synchronicity mechanisms, such as synchronous tactile stimulation on the body. These findings are particularly important not only because the sense of body ownership is a fundamental aspect of our bodily self-consciousness (Dijkerman, 2015; Blanke, Landis, Spinelli, \& Seeck, 2004) but also because the representation of one's own body is not fixed but rather the result of a predictive processing that is constantly updated by means of multisensory processes. Future studies should explore and extend these findings to clinical populations characterized by body image distortions and lack of bodily awareness, such as eating disorders.

\section{Acknowledgments}

The authors would like to thank Dora Tamari-Tutnjević, Mengbing Zhang, Amanda Hornsby, and Mariana von Mohr for assistance with data collection. They are grateful to Miss Catherine Maze for her help with the customized rubber hands. The study was supported by a European Research Council Starting Investigator Award [ERC-2012-STG GA313755] to A. K., a University of Hertfordshire studentship to L. C., and a Neuropsychoanalysis Foundation grant to L. C. Funding for the time of A. K. and L. C. has been partially provided by the Fund for Psychoanalytic Research through the American Psychoanalytic Association. The authors would like to thank the anonymous reviewers for their valuable comments and suggestions to improve the quality of the article.

Reprint requests should be sent to Laura Crucianelli, Department of Clinical, Educational and Health Psychology, University College London, 1-19 Torrington Place, London WC1E 7HB, United Kingdom, or via e-mail: 1.crucianelli@ucl.ac.uk.

\section{REFERENCES}

Abdulkarim, Z., \& Ehrsson, H. H. (2016). No causal link between changes in hand position sense and feeling of limb ownership in the rubber hand illusion. Attention. Perception. E Psychopbysics, 78, 707-720.

Ainley, V., \& Tsakiris, M. (2013). Body conscious? Interoceptive awareness, measured by heartbeat perception, is negatively correlated with self-objectification. PLoS One, 8, e 55568.

Amico, J. A., Tenicela, R., Johnston, J., \& Robinson, A. G. (1983). A time-dependent peak of oxytocin exists in cerebrospinal fluid but not in plasma of humans. Iournal of Clinical Endocrinologv \& Metabolism, 57, 947-951.

Apps, M. A., \& Tsakiris, M. (2014). The free-energy self: A predictive coding account of self-recognition. Neuroscience E Biobehavioral Reviews, 41, 85-97.

Bastos, A. M., Usrey, W. M., Adams, R. A., Mangun, G. R., Fries, P., \& Friston, K. J. (2012). Canonical microcircuits for predictive coding. Neuron, 76, 695-711.

Blanke, O. (2012). Multisensory brain mechanisms of bodily selfconsciousness. Nature Reviews Neuroscience, 13, 556-571.

Blanke, O., Landis, T., Spinelli, L., \& Seeck, M. (2004). Out-ofbody experience and autoscopy of neurological origin. Brain, 127, 243-258.

Born, J., Lange, T., Kern, W., McGregor, G. P., Bickel, U., \& Fehm, H. L. (2002). Sniffing neuropeptides: A transnasal approach to the human brain. Nature Neuroscience, 5 , 514-516.

Botvinick, M., \& Cohen, J. (1998). Rubber hands 'feel' touch that eyes see. Nature, 391, 756.

Buckingham, G., \& Goodale, M. A. (2013). Size matters: A single representation underlies our perceptions of heaviness in the size-weight illusion. PLoS One, 8, e54709.

Burbach, J. P., Young, L. J., \& Russell, J. (2006). Oxytocin: Synthesis, secretion and reproductive functions. In J. D. Neill (Ed.), Knobil and Neill's physiology of reproduction (3rd ed., pp. 3055-3128). London: Elsevier.

Cesari, P., \& Newell, K. M. (1999). The scaling of human grip configurations. Journal of Experimental Psychology: Human Perception and Performance, 25, 927-935.

Colonnello, V., \& Heinrichs, M. (2016). Oxytocin and selfconsciousness. Frontiers in Human Neuroscience, 10, 67.

Crucianelli, L., Krahé, C., Jenkinson, P. M., \& Fotopoulou, A. K. (2018). Interoceptive ingredients of body ownership: Affective touch and cardiac awareness in the rubber hand illusion. Cortex, 104, 180-192.

Crucianelli, L., Metcalf, N. K., Fotopoulou, A. K., \& Jenkinson, P. M. (2013). Bodily pleasure matters: Velocity of touch modulates body ownership during the rubber hand illusion. Frontiers in Psychology, 4, 703.

De Dreu, C. K. W., Greer, L. L., Handgraaf, M. J. J., Shalvi, S., Van Kleef, G. A., Bass, M., et al. (2010). The neuropeptide oxytocin regulates parochial altruism in intergroup conflict among humans. Science, 328, 1408-1411.

de Haan, A. M., Van Stralen, H. E., Smit, M., Keizer, A., Van der Stigchel, S., \& Dijkerman, H. C. (2017). No consistent cooling of the real hand in the rubber hand illusion. Acta Psychologica, 179, 68-77.

Deroy, O., Spence, C., \& Noppeney, U. (2016). Metacognition in multisensory perception. Trends in Cognitive Sciences, 20, 736-747.

Dijkerman, H. C. (2015). How do different aspects of selfconsciousness interact? Trends in Cognitive Sciences, 19, 427-428.

Ernst, M. O., \& Banks, M. S. (2002). Humans integrate visual and haptic information in a statistically optimal fashion. Nature, 415, 429-433.

Eshkevari, E., Rieger, E., Longo, M. R., Haggard, P., \& Treasure, J. (2012). Increased plasticity of the bodily self in eating disorders. Psychological Medicine, 42, 819-828.

Eshkevari, E., Rieger, E., Longo, M. R., Haggard, P., \& Treasure, J. (2014). Persistent body image disturbance following recovery from eating disorders. International Iournal of Eating Disorders, 47, 400-409.

Fairburn, C. G., \& Beglin, S. J. (1994). Assessment of eating disorders: Interview or self-report questionnaire? International Journal of Eating Disorders, 16, 363-370.

Fairburn, C. G., \& Beglin, S. J. (2008). Eating disorder examination questionnaire. In C. G. Faiburn (Ed.), Cognitive 
behaviour therapy and eating disorders (pp. 311-313). New York: Guilford Press.

Farmer, H., Tajadura-Jiménez, A., \& Tsakiris, M. (2012). Beyond the colour of my skin: How skin colour affects the sense of body-ownership. Consciousness and Cognition, $21,1242-1256$.

Farnè, A., Pavani, F., Meneghello, F., \& Ladavas, E. (2000). Left tactile extinction following visual stimulation of a rubber hand. Brain, 123, 2350-2360.

Feldman, H., \& Friston, K. (2010). Attention, uncertainty, and free-energy. Frontiers in Human Neuroscience, 4, 215.

Ferri, F., Chiarelli, A. M., Merla, A., Gallese, V., \& Costantini, M. (2013). The body beyond the body: Expectation of a sensory event is enough to induce ownership over a fake hand. Proceedings of the Royal Society of London, Series B, Biological Sciences, 280, 20131140.

Fiorillo, C. D., Tobler, P. N., \& Schultz, W. (2003). Discrete coding of reward probability and uncertainty by dopamine neurons. Science, 299, 1898-1902.

Fiser, J., Berkes, P., Orbán, G., \& Lengyel, M. (2010). Statistically optimal perception and learning: From behavior to neural representations. Trends in Cognitive Sciences, 14, 119-130.

Flanagan, J. R., \& Beltzner, M. A. (2000). Independence of perceptual and sensorimotor predictions in the size-weight illusion. Nature Neuroscience, 3, 737-741.

Fotopoulou, A., \& Tsakiris, M. (2017). Mentalizing homeostasis: The social origins of interoceptive inference.

Neuropsychoanalvsis, 19, 3-28.

Fredrickson, B. L., \& Roberts, T. A. (1997). Objectification theory: Toward understanding women's lived experiences and mental health risks. Psychologv of Women Ouarterly, 21, 173-206.

Fredrickson, B. L., Roberts, T. A., Noll, S. M., Quinn, D. M., \& Twenge, J. M. (1998). That swimsuit becomes you: Sex differences in self-objectification, restrained eating, and math performance. Lournal of Personality and Social Psychology, 75, 269-284.

Friston, K. (2005). A theory of cortical responses. Philosophical Transactions of the Royal Society, Series B, Biological Sciences, 360, 815-836.

Friston, K. (2008). Hierarchical models in the brain. PLoS Computational Biology, 4, e1000211.

Friston, K. (2010). The free-energy principle: A unified brain theory? Nature Reviews Neuroscience, 11, 127-138.

Friston, K., Adams, R. A., Perrinet, L., \& Breakspear, M. (2012). Perceptions as hypotheses: Saccades as experiments. Frontiers in Psychology, 3, 151.

Friston, K. J., \& Stephan, K. E. (2007). Free-energy and the brain. Synthese, 159, 417-458.

Gallagher, S. (2000). Philosophical conceptions of the self: Implications for cognitive science. Trends in Cognitive Science, 4, 14-21.

Gordon, I., Vander Wyk, B. C., Bennett, R. H., Cordeaux, C., Lucas, M. V., Eilbott, J. A., et al. (2013). Oxytocin enhances brain function in children with autism. Proceedings of the National Academv of Sciences. U.S.A., 110, 20953-20958.

Grinevich, V., Knobloch-Bollmann, H. S., Eliava, M., Busnelli, M., \& Chini, B. (2016). Assembling the puzzle: Pathways of oxytocin signaling in the brain. Biological Psychiatry, 79, 155-164.

Haggard, P., \& Jundi, S. (2009). Rubber hand illusions and size-weight illusions: Self-representation modulates representation of external objects. Perception, 38 , 1796-1803

Hosmer, D. W., Lemeshow, S., \& May, S. (2008). Model development. In D. Hosmer, S. Lemeshow, \& S. May (Eds.) Applied survival analysis: Regression modeling of time-to- event data (2nd ed., pp. 132-168). Hoboken, NJ: WileyInterscience.

Ide, M., \& Wada, M. (2017). Salivary oxytocin concentration associates with the subjective feeling of body ownership during the rubber hand illusion. Frontiers in Human Neuroscience, 11, 166.

Insel, T. R. (1992). Oxytocin-A neuropeptide for affiliation: Evidence from behavioral, receptor autoradiographic, and comparative studies. Psvchoneuroendocrinology, 17, 3-35.

Kagerbauer, S. M., Martin, J., Schuster, T., Blobner, M., Kochs, E. F., \& Landgraf, R. (2013). Plasma oxytocin and vasopressin do not predict neuropeptide concentrations in human cerebrospinal fluid. Journal of Neuroendocrinology, 25, 668-673.

Knill, D. C., \& Pouget, A. (2004). The Bayesian brain: The role of uncertainty in neural coding and computation. Trends in Neurosciences, 27, 712-719.

Leknes, S., Wessberg, J., Ellingsen, D.-M., Chelnokova, O., Olausson, H., \& Laeng, B. (2013). Oxytocin enhances pupil dilation and sensitivity to 'hidden' emotional expressions. Social Cognitive and Affective Neuroscience, 8, 741-749.

Leppanen, J., Ng, K. W., Tchanturia, K., \& Treasure, J. (2017). Meta-analysis of the effects of intranasal oxytocin on interpretation and expression of emotions. Neuroscience $\varepsilon$ Biobehavioral Reviews, 78, 125-144.

Limanowski, J., \& Blankenburg, F. (2015). Network activity underlying the illusory self-attribution of a dummy arm. Human Brain Mapping, 36, 2284-2304.

Longo, M. R., Schüür, F., Kammers, M. P., Tsakiris, M., \& Haggard, P. (2008). What is embodiment? A psychometric approach. Cognition, 107, 978-998.

Ludwig, M., \& Leng, G. (2006). Dendritic peptide release and peptide-dependent behaviours. Nature Reviews Neuroscience, 7, 126-136.

Ma, W. J., Beck, J. M., Latham, P. E., \& Pouget, A. (2006). Bayesian inference with probabilistic population codes. Nature Neuroscience, 9, 1432-1438.

MacDonald, E., Dadds, M. R., Brennan, J. L., Williams, K., Levy, F., \& Cauchi, A. J. (2011). A review of safety, sideeffects and subjective reactions to intranasal oxytocin in human research. Psychoneuroendocrinology, 36, 1114-1126.

MacDonald, K., \& MacDonald, T. M. (2010). The peptide that binds: A systematic review of oxytocin and its prosocial effects in humans. Harvard Review of Psychiatry, 18, 1-21.

Martinaud, O., Besharati, S., Jenkinson, P. M., \& Fotopoulou, A. (2017). Ownership illusions in patients with body delusions: Different neural profiles of visual capture and disownership. Cortex, 87, 174-185.

McGlone, F., Olausson, H., Boyle, J. A., Jones-Gotman, M., Dancer, C., Guest, S., et al. (2012). Touching and feeling: Differences in pleasant touch processing between glabrous and hairy skin in humans. European Iournal of Neuroscience, 35, 1782-1788.

Miner-Rubino, K., Twenge, J. M., \& Fredrickson, B. L. (2002). Trait self-objectification in women: Affective and personality correlates. Lournal of Research in Personality, 36, 147-172.

Mond, J. M., Myers, T. C., Crosby, R. D., Hay, P. J., Rodgers, B., Morgan, J. F., et al. (2008). Screening for eating disorders in primary care: EDE-Q versus SCOFF. Behaviour Research and Therapy, 46, 612-622.

Moseley, G. L., Olthof, N., Venema, A., Don, S., Wijers, M., Gallace, A., et al. (2008). Psychologically induced cooling of a specific body part caused by the illusory ownership of an artificial counterpart. Proceedings of the National Academy of Sciences, U.S.A., 105, 13169-13173.

O'Reilly, J. X., Jbabdi, S., \& Behrens, T. E. (2012). How can a Bayesian approach inform neuroscience? European Iournal of Neuroscience, 35, 1169-1179. 
Paloyelis, Y., Doyle, O. M., Zelaya, F. O., Maltezos, S., Williams, S. C., Fotopoulou, A., et al. (2016). A spatiotemporal profile of in vivo cerebral blood flow changes following intranasal oxytocin in humans. Biological Psychiatry, 79, 693-705.

Paloyelis, Y., Krahé, C., Maltezos, S., Williams, S. C., Howard, M. A., \& Fotopoulou, A. (2016). The analgesic effect of oxytocin in humans: A double-blind, placebo-controlled cross-over study using laser-evoked potentials. Journal of Neuroendocrinology, 28. doi:10.1111/jne.12347.

Panagiotopoulou, E., Filippetti, M. L., Tsakiris, M., \& Fotopoulou, A. (2017). Affective touch enhances self-face recognition during multisensory integration. Scientific Reports, 7, 12883.

Pavani, F., Spence, C., \& Driver, J. (2000). Visual capture of touch: Out-of-the-body experiences with rubber gloves. Psvchological Science, 11, 353-359.

Peterson, C. B., Crosby, R. D., Wonderlich, S. A., Joiner, T., Crow, S. J., Mitchell, J. E., et al. (2007). Psychometric properties of the eating disorder examination-questionnaire: Factor structure and internal consistency. International Iournal of Eating Disorders, 40, 386-389.

Preston, C., \& Ehrsson, H. H. (2014). Illusory changes in body size modulate body satisfaction in a way that is related to non-clinical eating disorder psychopathology. PLoS One, 9, e85773.

Quattrocki, E., \& Friston, K. (2014). Autism, oxytocin and interoception. Neuroscience \& Biobehavioral Reviews, 47, 410-430.

Rao, R. P., \& Ballard, D. H. (1999). Predictive coding in the visual cortex: A functional interpretation of some extra-classical receptive-field effects. Nature Neuroscience, 2, 79-87.

Rocchetti, M., Radua, J., Paloyelis, Y., Xenaki, L. A., Frascarelli, M., Caverzasi, E., et al. (2014). Neurofunctional maps of the 'maternal brain' and the effects of oxytocin: A multimodal voxel-based meta-analysis. Psvchiatrv and Clinical Neurosciences, 68, 733-751.

Rohde, M., Di Luca, M., \& Ernst, M. O. (2011). The rubber hand illusion: Feeling of ownership and proprioceptive drift do not go hand in hand. PLoS One, 6, e21659.

Rohe, T., \& Noppeney, U. (2015). Cortical hierarchies perform Bayesian causal inference in multisensory perception. PLoS Biology, 13, e1002073.

Salonia, A., Nappi, R. E., Pontillo, M., Daverio, R., Smeraldi, A., Briganti, A., et al. (2005). Menstrual cycle-related changes in plasma oxytocin are relevant to normal sexual function in healthy women. Hormones and Behavior, 47, 164-169.

Samad, M., Chung, A. J., \& Shams, L. (2015). Perception of body ownership is driven by Bayesian sensory inference. PLoS One, 10, e0117178.

Shamay-Tsoory, S. G., \& Abu-Akel, A. (2016). The social salience hypothesis of oxytocin. Biological Psvchiatrv, 79, 194-202.

Shipp, S., Adams, R. A., \& Friston, K. J. (2013). Reflections on agranular architecture: Predictive coding in the motor cortex. Trends in Neurosciences, 36, 706-716.

Stoop, R. (2012). Neuromodulation by oxytocin and vasopressin. Neuron, 76, 142-159.

Tiggemann, M., \& Lynch, J. E. (2001). Body image across the life span in adult women: The role of self-objectification. Developmental Psvchologv, 37, 243-253.

Treasure, J., \& Schmidt, U. (2013). The cognitive-interpersonal maintenance model of anorexia nervosa revisited: A summary of the evidence for cognitive, socio-emotional and interpersonal predisposing and perpetuating factors. Journal of Eating Disorders, 1, 13.

Tsakiris, M. (2010). My body in the brain: A neurocognitive model of body-ownership. Neuropsvchologia, 48, 703-712.

Tsakiris, M., \& Haggard, P. (2005). The rubber hand illusion revisited: Visuotactile integration and self-attribution. Journal of Experimental Psychology: Human Perception and Performance, 31, 80-91.

Uvnäs-Moberg, K., Handlin, L., \& Petersson, M. (2015). Selfsoothing behaviors with particular reference to oxytocin release induced by non-noxious sensory stimulation. Frontiers in Psvchology, 5, 1529.

Wagner, A., Barbarich-Marsteller, N. C., Frank, G. K., Bailer, U. F., Wonderlich, S. A., Crosby, R. D., et al. (2006). Personality traits after recovery from eating disorders: Do subtypes differ? International Journal of Eating Disorders, 39, 276-284.

Yu, A. J., \& Dayan, P. (2005). Uncertainty, neuromodulation, and attention. Neuron, 46, 681-692.

Zeller, D., Friston, K. J., \& Classen, J. (2016). Dynamic causal modeling of touch-evoked potentials in the rubber hand illusion. Neuroimage, 138, 266-273.

Zeller, D., Litvak, V., Friston, K. J., \& Classen, J. (2014). Sensory processing and the rubber hand illusion-An evoked potentials study. Journal of Cognitive Neuroscience, 27, $573-582$. 